\title{
Usefulness of the Bidirectional Glenn Procedure as Staged Reconstruction for the Functional Single Ventricle
}

\author{
Ara K. Pridjian, MD, Alan M. Mendelsohn, MD, Flavian M. Lupinetti, MD, \\ Robert H. Beekman III, MD, Macdonald Dick II, MD, \\ Gerald Serwer, MD, and Edward L. Bove, MD
}

The bidirectional Clenn operation may be particularly useful as an intermediate procedure before Fontan correction in highrisk patients. From 0ctober 1989 through Fobruary 1992, 50 patients 1 to 60 months old (median 12) have undergone a bidirectional Glenn operation. Diagnoses included hypoplastic left heart syndrome in 21 patients, pulmonary atresia with intact ventricular septum in 10, tricuspid valve atresia in 9, other complex univentricular heart defects in 9, and Ebstein's anomaly in 1. Mean pulmonary vascular resistance was $2.2 \pm 0.2$ Wood U (range 0.5 to 7.3) and mean pulmonary artery area Nakata index was $318 \pm \mathrm{mm}^{2} / \mathrm{m}^{2}$ (range 80 to 821 ). Additional procedures were performed in 17 patients, including pulmonary artery reconstruction in 15 (29\%) and bilateral caval anastomoses in 5 (10\%). There wore 4 hospital deaths (8\%). Two deaths resulted from myocardial infarction in patients with pulmonary atresia with intact ventricular septum and sinusoids and 1 from severe pulmonary vascular disease in a patient with hypoplastic left heart syndrome. There was 1 late death from pneumonia. Actuarial survival is $92 \pm 4 \%$ at 1 month and beyond, with a mean follow-up of $13.4 \pm 1$ months. Risk factor analysis showed that pulmonary vascular resistance $>3$ Wood $U$ and pulmonary arteny distortion were associated with increased mortality. Twelve patients have undergone a Fontan procedure at a mean duration after bidirectional Glenn of 18 months with 1 death (8\%). The bidirectional Glenn procedure provides excellent palliation in high-risk patients and appears useful as a staging procedure before Fontan correction.

(Am J Cardiol 1993;71:959-962)

From The Department of Surgery, Section of Thoracic Surgery and The Department of Pediatrics, Division of Pediatric Cardiology, C.S. Mott Children's Hospital, The University of Michigan School of Medicine, Ann Arhor, Michigan. Manuscript received September 24, 1992; revised manuscript received and accepted November 6, 1992.

Address for reprints: Edward L. Bove, MD, 2120 Taubman Center, Box 0344, 1500 East Medical Center Drive, Ann Arbor, Michigan 48109.
$\mathrm{T}$ he Fontan principle has been applied for the treatment of patients with increasingly complex forms of congenital heart disease. Most high-risk patients require a variety of palliative operations early in life. Accordingly, it is important at the time of these palliative operations to minimize conditions known to increase the risk for the Fontan operation. Some conditions that increase this risk include pulmonary artery distortion, elevated pulmonary vascular resistance, pulmonary hypertension, poor ventricular function, atrioventricular valve regurgitation, complex anatomy and young age. ${ }^{1-3}$ Palliative procedures that result in increased pressure or volume overload on the systemic ventricular chamber are likely to diminish the chances of a successful Fontan. ${ }^{4}$ The bidirectional Glenn procedure directs superior vena cava blood to the undivided pulmonary arteries and can provide satisfactory systemic oxygen saturation without ventricular volume overload. This procedure has received increased attention recently as a potentially useful intermediate step, often combined with additional reconstructive procedures, in high-risk patients before the Fontan operation. ${ }^{5-7}$ This report reviews our experience and interim observations in 50 patients who underwent the bidirectional Glenn procedure at the University of Michigan Medical Center. Early- and intermediate-term results were analyzed to assess operative risk, adequacy of palliation and suitability for Fontan correction.

\section{METHODS}

Patients: The records of 50 consecutive infants and children undergoing the bidirectional Glenn procedure from October 1989 through February 1992, at C.S. Mott Children's Hospital of The University of Michigan were reviewed. There were 27 men and 23 women ranging in age from 1 to 60 months (median 12). Diagnoses were hypoplastic left heart syndrome in 21 patients, pulmonary atresia with intact ventricular septum in 10 , tricuspid atresia in 9, double-inlet left ventricle in 5, heterotaxy syndrome in 4 and Ebstein's anomaly in 1. Four of the 10 patients with pulmonary atresia and intact ventricular septum had significant right ventricular coronary connections. All patients underwent complete preoperative evaluation including 2-dimensional and Doppler echocardiography and cardiac catheterization. The pulmonary artery index was determined by the method of Nakata et al. ${ }^{8}$ Pulmonary arteries were considered hypoplastic if this index was $<200 \mathrm{~mm}^{2} / \mathrm{m}^{2}$. 


\begin{tabular}{|c|c|c|}
\hline Risk Factor & $\begin{array}{l}\text { Mortality (\%) } \\
\text { if Present }\end{array}$ & $\begin{array}{c}\mathrm{p} \\
\text { Value }\end{array}$ \\
\hline Age $<1$ year & $3 / 24(13 \%)$ & NS \\
\hline Nakata index $<200 \mathrm{~mm}^{2} / \mathrm{m}^{2}$ & $2 / 14(14 \%)$ & NS \\
\hline \multicolumn{3}{|l|}{ Diagnosis } \\
\hline Hypoplastic left heart syndrome & $1 / 21(5 \%)$ & NS \\
\hline $\begin{array}{l}\text { Pulmonary atresia with intact ventricular } \\
\text { septum }\end{array}$ & $2 / 10(20 \%)$ & NS \\
\hline Tricuspid atresia & $1 / 9(11 \%)$ & NS \\
\hline Double-inlet left ventricle & $0 / 5(0 \%)$ & NS \\
\hline Heterotaxy syndrome & $0 / 4(0 \%)$ & NS \\
\hline Ebstein's anomaly & $0 / 1(0 \%)$ & NS \\
\hline Ejection fraction $\leq 50 \%$ & $3 / 15(20 \%)$ & NS \\
\hline $\begin{array}{l}\text { Concomitant operative procedure (excluding } \\
\text { PA reconstruction) }\end{array}$ & $1 / 17(6 \%)$ & NS \\
\hline PA reconstruction & $4 / 15(27 \%)$ & 0.006 \\
\hline Pulmonary vascular resistance $>3$ Wood $U$ & $3 / 7(43 \%)$ & 0.005 \\
\hline
\end{tabular}

Patients with hypoplastic left heart syndrome generally underwent the bidirectional Glenn procedure between 4 and 6 months of age as part of a protocol of staged repair leading to Fontan correction. ${ }^{9}$ Patients with pulmonary atresia and intact ventricular septum who were not candidates for a 2-ventricle repair underwent a bidirectional Glenn procedure as intermediate palliation before Fontan. Patients in all other anatomic subsets underwent operation when significant symptoms were present, most often progressive hypoxemia or exercise intolerance. In each, at least 1 significant risk factor for the Fontan procedure was present. These risk factors included age $<1$ year in 27 patients, significant pulmonary artery distortion in 15 , hypoplastic pulmonary arteries (Nakata index $<200 \mathrm{~mm}^{2} / \mathrm{m}^{2}$ ) in 14, ejection fraction $<50 \%$ in 12 , pulmonary vascular resistance $>3$ Wood U in 7 , and significant atrioventricular valve regurgitation requiring repair in 2 .

Forty-seven patients (94\%) underwent prior palliative procedures including 44 Blalock-Taussig shunts and 3 pulmonary artery bands. Fifteen of the Blalock-Taussig shunts were performed as isolated procedures and 29 were performed as part of other palliative procedures. Twenty-one concomitant procedures were performed in 17 patients. These included 11 atrial septectomies, 3 tricuspid valve excisions, 2 atrioventricular valve repairs, 2 outlet foramen enlargements, 1 Damus-Kaye-Stansel connection, 1 lung biopsy and 1 pacemaker insertion. Five bilateral bidirectional Glenn procedures were performed in patients with bilateral superior vena cavae. Fifteen patients with pulmonary artery distortion underwent pulmonary artery reconstruction with bidirectional Glenn. Of these, 12 received patch angioplasty and 3 received interposition conduits between the right and left pulmonary arteries.

Surgical technique: All operations were performed using cardiopulmonary bypass with mild to moderate hypothermia. A single cannula was placed in the right atrium for venous return and the superior vena cava was temporarily occluded during the anastomosis. If central venous pressure became significantly elevated during superior vena cava occlusion, a small cannula was in- serted in the innominate vein for decompression. Anastomosis of the superior vena cava to the pulmonary artery was performed with continuous polypropylene suture for the posterior half of the suture line, and interrupted polypropylene sutures for the anterior half to prevent anastomotic narrowing. In 19 patients, the divided cardiac side of the superior vena cava was anastomosed to the inferior surface of the right pulmonary artery and an intraatrial polytetrafluoroethylene patch was then placed to close the internal orifice of the superior vena cava.

Data analysis: All data were analyzed using SAS software. Survival was determined using standard actuarial techniques. ${ }^{10}$ The following risk factors were examined by both univariate (Fisher exact and Student's $t$ test) and multivariate linear regression methods to determine their effect on survival: age, diagnosis, pulmonary vascular resistance, pulmonary artery reconstruction, Nakata index, concomitant procedures and ejection fraction. Age, pulmonary vascular resistance and ejection fraction were examined as both categorical and continuous variables. A Student's $t$ test was used to determine differences between preoperative, postoperative and follow-up oxygen saturations. All values are reported as mean $\pm \mathrm{SE}$ unless otherwise specified. The $70 \%$ confidence limits were calculated. A p value $<0.05$ was considered significant.

\section{RESULTS}

Survival: There were 4 hospital deaths $(8 \%, 70 \%$; confidence limits, 4 to $12 \%$ ). Two resulted from myocardial ischemia in patients with pulmonary atresia and intact ventricular septum and significant right ventricular coronary connections, and 1 resulted from severe pulmonary vascular disease in a patient with hypoplastic left heart syndrome and marked pulmonary artery distortion. There was 1 late death due to pneumonia caused by respiratory syncytial virus. Actuarial survival was $92 \pm 4 \%$ at 1 month and beyond. Risk factor analysis (Table I) failed to demonstrate an increased risk for death related to age, diagnosis, pulmonary artery hypoplasia, ejection fraction, or concomitant procedures other than pulmonary artery reconstruction. However, pulmonary vascular resistance $>3$ Wood $U$ and the need for pulmonary artery reconstruction did predict increased operative mortality. After univariate analysis all risk factors were entered into a stepwise multiple logistic analysis which failed to identify any additional risk factors, alone or in combination, that predicted increased risk of death.

Oxygen saturation: Oxygen saturation was $77 \pm 6 \%$ at preoperative cardiac catheterization, $79 \pm 5 \%$ just before hospital discharge, and $80 \pm 6 \%$ at the time of most recent follow-up. A significant difference was found between preoperative and late follow-up oxygen saturations ( $p<0.05$ ), but not between preoperative and hospital discharge values. Two patients remained severely cyanotic after the bidirectional Glenn procedure with oxygen saturations of 61 and $62 \%$. These patients had pulmonary vascular resistances of 7.3 and 2.6 Wood $U$ and received $4 \mathrm{~mm}$ systemic to pulmonary artery shunts with improvement in oxygen saturation to 70 and $78 \%$, respectively. 
Morbidity: Major postoperative complications included prolonged pleural effusions in 2 patients. Conservative measures were eventually successful in 1 patient. In the other, thoracotomy with pleurodesis and thoracic duct ligation were necessary. One patient sustained a left phrenic nerve palsy. A left-sided cerebrovascular accident occurred in 1 patient who has a residual minor neurologic deficit. One patient with profound hypoxemia after the bidirectional Glenn procedure underwent reoperation to ligate a previously undiagnosed left superior vena cava.

Fontan procedure: To date, 12 patients have undergone the Fontan operation at a mean of 18 months after the bidirectional Glenn procedure, with 1 death $(8 \%)$. Their diagnoses were hypoplastic left heart syndrome in 7 , pulmonary atresia with intact ventricular septum in 2 , tricuspid atresia in 2 and double-inlet left ventricle in 1. Risk factors present at the time of bidirectional Glenn procedure and those present at the time of Fontan are listed in Table II. Pulmonary artery reconstruction was not required in any patient at the time of the Fontan procedure. Prolonged pleural effusions developed in 2 patients.

\section{DISCussion}

The results of this experience suggest that although the bidirectional Glenn procedure can be safely performed in most patients who otherwise represent a high risk for the Fontan operation, pulmonary artery distortion and pulmonary vascular resistance in excess of 3 Wood U are significant risk factors for death. To decrease the risk of the bidirectional Glenn procedure, careful attention to the timing and choice of preliminary palliative procedures should favorably influence the incidence of significant pulmonary artery distortion and pulmonary vascular disease.

Implications for initial and subsequent pallia tion: When pulmonary overcirculation exists in patients with a functional single ventricle, disconnection of the main pulmonary artery and restriction of pulmonary blood flow through a limited systemic to pulmonary shunt are recommended early in life. Systemic to pulmonary artery shunts may cause distortion of the pulmonary arteries, and the ventricular volume overload that results from these shunts may produce ventricular dysfunction. ${ }^{4,11}$ For these reasons, we prefer the bidirectional Glenn procedure rather than placement of an arterial shunt in patients beyond the neonatal period who are not otherwise candidates for a Fontan procedure.

Initial palliation for patients with functional single ventricle and pulmonary hypoperfusion is usually accomplished with a $5 \mathrm{~mm}$ polytetrafluoroethylene systemic to pulmonary artery shunt. Shunts of this size are well-tolerated by most patients and provide effective palliation for at least 1 year. In patients with hypoplastic left heart syndrome, however, the balance between the pulmonary and systemic circulations is more delicate, especially in the early postoperative period. Although oxygen saturation is lower, use of 3.5 or $4.0 \mathrm{~mm}$ shunts has been associated with more stable postoperative hemodynamics after the Norwood procedure. However, cyanosis tends to progress more rapidly with such small
TABLE ॥ Risk Factors Present at Time of Bidirectional Glenn Procedure and at Time of Subsequent Fontan Operation Among 12 Patients Undergoing Both Procedures

\begin{tabular}{|lcc|}
\hline \multicolumn{1}{|c|}{ Risk Factor } & $\begin{array}{c}\text { No. with Factor } \\
\text { Present at Time of } \\
\text { Bidirectional Glenn }\end{array}$ & $\begin{array}{c}\text { No. with Factor } \\
\text { Present at } \\
\text { Time of Fontan }\end{array}$ \\
\hline Age $<1$ year & 4 & 0 \\
Nakata index $<200 \mathrm{~mm}^{2} / \mathrm{m}^{2}$ & 3 & 0 \\
Ejection fraction $\leq 50 \%$ & 3 & 2 \\
Pulmonary artery distortion & 3 & 0 \\
Atrioventricular valve insuffi- & 2 & 0 \\
ciency & 3 & 2 \\
Pulmonary vascular resistance & 3 & \\
$>2$ Wood U & & \\
\hline
\end{tabular}

shunts, and the bidirectional Glenn procedure may be required at an earlier age.

Implications for ventricular function: The bidirectional Glenn procedure should be performed early in life to reduce the volume load on the functional single ventricle and improve ventricular function. We have avoided pulmonary artery banding in these patients because this may result in progressive ventricular hypertrophy from obstruction of the outlet foramen in hearts with a subaortic chamber. Furthermore, we prefer to remove all other sources of pulmonary blood flow, since the bidirectional Glenn alone results in a satisfactory systemic saturation in nearly all patients, reserving the addition of a small shunt for those with unacceptable hypoxemia.

Implications for patients with hypoplantic left heart syndrome or pulmonary atresia with intact ventricular septum: Because our previous experience with either early $(<1$ year) Fontan procedures or placement of an additional systemic to pulmonary artery shunt has proven unsatisfactory in patients with hypoplastic left heart syndrome, the bidirectional Glenn procedure was added as the second stage of the reconstruction. ${ }^{9}$ Similarly, because of unsatisfactory results achieved in patients with pulmonary atresia with intact ventricular septum, patients with this diagnosis who were not candidates for a 2-ventricle repair have undergone a bidirectional Glenn procedure as intermediate palliation before Fontan. This may be particularly important when significant right ventricular coronary connections are present. These connections occur in $63 \%$ of patients with this condition, and coronary artery stenoses with right ventriculardependent coronary blood flow may be found in approximately $15 \%$ of these..$^{12}$ If these patients are otherwise acceptable candidates for ultimate Fontan, we perform a bidirectional Glenn procedure at approximately 4 to 6 months of age. The Fontan is accomplished approximately 1 year later using the lateral intraatrial tunnel modification which allows fully saturated pulmonary venous blood to enter the right ventricle and coronary arteries. ${ }^{13}$ With the advent of the fenestrated Fontan, indications for a bidirectional Glenn procedure over an early Fontan may be in a state of flux. However, we think that patients in these 2 anatomic subsets in particular are managed equally well or better by intermediate bidirectional Glenn procedure.

Implications for patients with increased pulmonary vascular resistance: Although elevated pulmonary vas- 
cular resistance and pulmonary artery distortion do not preclude a successful bidirectional Glenn procedure, our data suggest that the risk of mortality is high when the pulmonary vascular resistance is $>3$ Wood $U$ or when there is significant pulmonary artery distortion requiring reconstruction. If cyanosis remains severe after a bidirectional Glenn, a small supplemental systemic to pulmonary artery shunt may be added, as in the 2 patients in this series. The hemodynamic effects of pulsatile pulmonary How with a bidirectional Glenn procedure have been studied by Matsuda, and the safety and efficacy of this tcchnique have been demonstrated. ${ }^{14}$

Technical considerations: Anastomosis of both ends of the divided superior vena cava to the right pulmonary artery with placement of a prosthetic patch over the internal orifice of the superior vena cava is a technical modification similar in purpose to the "hemifontan" procedure described by Douville et al. 15 As the inferior end of the cavopulmonary connection is already made, all that remains at the time of Fontan is to remove the intraatrial patch and place the lateral intraatrial tunnel. To date, 3 patients in our series with this modification have undergone the Fontan. The inferior cavopulmonary connection was of adequate size in 2 and there was no thrombus in the "blind" limb between the pulmonary artery and the patch. We chose to use cardiopulmonary bypass in all patients in order to optimize exposure for the anastomoses and support systemic oxygenation since all other sources of pulmonary blood flow were interrupted.

In conclusion, the bidirectional Glenn procedure is a useful operation that results in effective palliation without ventricular volume overload. Furthermore, it provides the setting in which to ameliorate anatomic and physiologic factors that increase Fontan risk and is par- ticularly beneficial for the young infant with complex congenital heart disease.

1. Choussat A, Fontan F, Besse P, Vallot F, Chauve A, Bricaud H. Selection criteria for Fontan's procedure. In: Anderson FH, Shinebourne EA, eds. Paediatric Cardiology. Edinburgh: Churchill Livingstone, 1978:559-566.

2. Stefanelli B, Kirklin JW, Naftel DC, Blackstone EH, Pacifico AD, Kirklin JK, Soto B, Bargeron LM Jr. Early and intermediate-term (10 year) results of surgery for univentricular atrioventricular connection ("single ventricle"). Am J Cardiol 1984;54:811-821.

3. Kirklin JK, Blackstone EH, Kirklin JW, Pacifico AD, Bargeron LM Jr. The Fontan operation. Ventricular hypertrophy, age, and date of operation as risk factors. J Thorac Cardiovasc Surg 1986;92:1049-1064.

4. Mietus-Snyder M, Lang P, Mayer JE, Jonas RA, Castaneda AR, Lock JE. Childhood systemic-pulmonary shunts: subsequent suitability of Fontan operation. $\mathrm{Cir}$ culation 1987;76(suppl III):III-39-III-44.

5. Lamberti JJ, Spicer RL, Waldman JD, Grehl TM, Thomson D, George L, Kirkpatrick SE, Mathewson JW. The bidirectional cavopulmonary shunt. $J$ Thorac Cardiovasc Surg 1990;100:22-30.

6. Hopkins RA, Armstrong BE, Serwer GA, Peterson RJ, Oldham HN Jr. Physiological rationale for a bidirectional cavopulmonary shunt. $J$ Thorac Cardiovasc Surg 1985;90:391-398.

7. Bridges ND, Jonas RA, Mayer JE, Flanagan MF, Keane JF, Castaneda AR. Bidirectional cavopulmonary anastomosis as interim palliation for high-risk Fontan can didates. Carly results. Circulation 1990;82(suppl IV):IV-170-IV-176.

8. Nakata S, Imai Y, Takanashi Y, Kurosawa H, Tezuka K, Nakazawa M, Ando $M$, Takao A. A new method for the quantitative standardization of cross-sectional areas of the pulmonary arteries in congenital heart diseases with decreased pulmonary blood flow. $J$ Thorac Cardiovasc Surg 1984;88:610-619.

9. Norwood WI. Hypoplastic left heart syndrome. Cardiol Clin 1989;7:377-385.

10. Kaplan EL, Meier P. Nonparametric estimation from incomplete observations. $J$ Am Stat Assoc 1958;53:457-481.

11. Graham TP Jr, Franklin RCG, Wyse RKH, Gooch V, Deanfield JE. Left ventricular wall stress and contractile function in childhood: normal values and comparison of Fontan repair versus palliation only in patients with tricuspid atresia. Circulation 1986;74(suppl I):I-61-I-69.

12. Calder LA, Co EE, Sage MD. Coronary arterial abnormalities in pulmonary atresia with intact ventricular septum. Am $J$ Cardiol 1987;59:436-442.

13. Pearl JM, Laks H, Stein DG, Drinkwater DC, George BL, Williams RG. Total cavopulmonary anastomosis versus conventional modified Fontan procedure. Ann Thorac Surg 1991;52:189 196.

14. Kobayashi J, Matsuda $H$, Nakano S, Shimazaki $Y$, Ikawa S, Mitsuno M, Takahashi Y, Kawashima Y, Arisawa J, Matsushita T. Hemodyanamic effects of bidirectional cavopulmonary shunt with pulsatile pulmonary flow. Circulation 1991, 84(suppl III):III-219-III-225.

15. Douville EC, Sade RM, Fyfe DA. Hemi-Fontan operation in surgery for single ventricle: a preliminary report. Ann Thorac Surg 1991;51:893-900. 